\title{
The Pain of Ankylosing Spondylitis
}

ARMIN E. GOOD, M.D.

$T^{n}$ he pain of ankylosing spondylitis differs according to the stage of disease. In actual practice, however, these stages may overlap somewhat, especially in patients with accelerated disease.

Stage I. Isolated sacroiliac pain is sometimes reported, although most patients seek medical attention only later, when the spine becomes symptomatic. Early sacroiliac inflammation is nearly always referred to as "hip pain," but when pressed the patient generally points to the outer quadrant of the buttock. Pain usually alternates from side to side, but is sometimes persistently unilateral, occasionally with referral into the thigh as far as the knee. This pain is often mislabeled as "sciatica" and may be exacerbated by the straight leg raising test.

"Pure" sacroiliac pain is more often mild and nagging than severe, but nevertheless has a propensity for awakening the patient at night.

Pain is ameliorated by getting up, sitting, or walking about. In fact, the most important screening question for ankylosing spondylitis is: "Does the pain awaken you at night and get you out of bed?" A few patients have transient sharp and severe pain, exacerbated by jarring or vigorous activity.

The sacroiliac syndrome often starts during adolescence and generally persists less than a year, followed by an extended asymptomatic period.

Stage II. The prolonged middle phase of ankylosing spondylitis provides the main indication for sustained antiinflammatory and analgesic drug therapy. When the disease starts low in the back, the initial pain is limited to the lumbar segment. However, a considerable minority of patients initially complain of simultaneous or even exclusive interscapular or low cervical pain. Whatever the site, the pain is largely "inflammatory" rather than "mechanical" and is not relieved by rest or immobility.

Crippling pain and stiffness is frequently described immediately after getting up in the morning, only to disappear as the stiffness resolves within $\mathbf{3 0}$ to 90 minutes. Most often, pain breaks through again by mid-afternoon if the patient attempts to sustain a normal day's work.

As with sacroiliac pain, spinal pain varies in severity. Many patients with spondylitis have a vigorous, athletic life-style before and during their early disease. In others constant pain, hypochondriasis, and depression develop, suggesting a psychogenic component.

During the course of ankylosing spondylitis, different spinal areas may become painful, often superseding one another or skipping back and forth. Minor flares can last for days or weeks, and are sometimes apparently related to trauma, such as a mild fall. Of special interest is the presence of anterior chest pain experienced by a large number of patients, however briefly, at some point during the illness. This chest pain is generally described as nonanginal and "mechanical"- - typically a sharp, jabbing pain encircling the hemithorax precipitated by coughing or laughing. If often coexists with dorsal spine pain, but can also appear as an island of pain over the precordium in patients unaware of dorsal involvement. Anterior chest pain may be referred from inflamed costospinal and costotransverse joints, or it may originate directly from anterior sites, such as the manubriosternal and sternoclavicular joints.

Stage III. In the late period of disease, patients are free of morning stiffness and pain at rest, but complain of nagging interscapular, neck, and even low back pain. These patients nearly always have rigid spines, dorsal kyphosis,

From the Rheumatology Section, Ann Arbor Veterans Administration Medical Center, and the Department of Medicine, University of Michigan. Requests for reprints should be addressed to Dr. Armin E. Good, Rheumatology Section, Veterans Administration Medical Centor, 2215 Fuller Road, Ann Arbor, Michigan 48205. 
and forward protrusion of the head, and must maintain postural muscle contraction when upright to counteract the force of gravity. Pain is relieved promptly by lying down and relaxing stressed muscles. True muscle spasm is not seen.

When excruciating focal pain appears abruptly in patients with late or dormant ankylosing spondylitis, a pseudoarthrosis should be suspected. Most of these patients have ankylosed dorsal and lumbar truncal segments, which finally give way, usually at a fulcrum in the lower dorsal spine. Severe pain resolves only with immobilization and reankylosis.

Drug Therapy. Aspirin may still be a reasonable first choice drug, especially for patients in stage I or II. When aspirin is not adequate, phenylbutazone and indomethacin have traditionally been used to control pain. Despite their improved effectiveness over aspirin, these agents are associated with a generally high incidence of gastrointestinal side effects. Phenylbutazone carries with it a risk of marrow aplasia and must be monitored by periodic blood cell counts. Indomethacin-related headache and depressive reactions are not infrequent. A new agent, flurbiprofen, appears to offer both excellent analgesic efficacy and a low side-effect profile for the treatment of ankylosing spondylitis. Two recent studies confirming the efficacy and safety of flurbiprofen compared with phenylbutazone and indomethacin are described in the following articles. 